\title{
Chapter 19 \\ The Pale Grass Blue Butterfly \\ as an Indicator for the Biological Effect \\ of the Fukushima Daiichi Nuclear Power \\ Plant Accident
}

\author{
Joji M. Otaki
}

\begin{abstract}
The pale grass blue butterfly (Zizeeria maha) has been used as an indicator for the biological effect of the Fukushima Daiichi Nuclear Power Plant (FNPP) since 2011. Various biological aspects have been examined that point out the biological impact of the FNPP accident through the morphological abnormalities and death of the butterfly. However, the mechanisms responsible for such biological effect in the contaminated field remain elusive. In this article, previous and current studies on the pale grass blue butterfly in response to the FNPP accident are concisely reviewed to provide new directions for answering questions about the field effect.
\end{abstract}

Keywords Fukushima Daiichi Nuclear Power Plant accident · Field effect · Field-laboratory paradox $\cdot$ Low-dose exposure $\cdot$ Pale grass blue butterfly

\subsection{Introduction}

The pale grass blue butterfly (Zizeeria maha) is a common butterfly found in humanized environments throughout Japan (except Hokkaido, the northernmost major island). Larvae of this butterfly basically eat only one weed species, Oxalis corniculata, which is common in various environments including agricultural villages and metropolitan cities. The pale grass blue butterfly overwinters as larvae, the larvae pupate in the early spring, and adult butterflies come out at the end of April or beginning of May. Its life cycle is complete in approximately a month, and the butterfly produces several generations per year.

We chose this butterfly to study color pattern development and evolution many years before the Fukushima Daiichi Nuclear Power Plant (FNPP) accident occurred

\footnotetext{
J. M. Otaki $(\bowtie)$

The BCPH Unit of Molecular Physiology, Department of Chemistry, Biology and Marine Science, Faculty of Science, University of the Ryukyus, Okinawa, Japan

e-mail: otaki@sci.u-ryukyu.ac.jp
} 
in 2011. We established a standard rearing method [1] and studied a phenotypically plastic aspect of the wing color patterns as an important field case of "real-time" evolution in response to environmental changes $[2,3]$. The color pattern changes (called modifications) that are induced in response to cold shock and observed in the northern range-margin population (Fukaura, Aomori Prefecture) have been established as phenotypically plastic traits in response to environmental changes and their genetic assimilation in the population [2-5]. In contrast, color pattern changes (called aberrations or abnormalities) that are different from modifications can also be produced by introducing genetic mutations experimentally [6]. Both cases are employed to understand the mechanisms of color pattern development in this butterfly [7].

Following the FNPP accident, we decided to apply this butterfly system to probe the biological impact of the FNPP accident [8]. We recently demonstrated quantitatively that the pale grass blue butterfly is an excellent indicator for human-living environments in terms of sampling efficiency [9]. In this article, our findings are summarized, and new directions for answering emerging questions are presented. Because of a strict page limitation, we have almost exclusively focused on our own studies and are unable to refer to other important contributions to this field.

\subsection{Three Research Objectives}

The first objective of our research is to clarify the presence and the cause of the biological aberration that followed the FNPP accident. Because any biological aberration might have been caused by multiple factors, the causal demonstration is not straightforward. We referred to the Koch's postulate for infectious diseases and proposed the postulates of pollutant-induced biological impacts [10]. The postulates are a set of six criteria that must be met to demonstrate the effect of the pollutants in question (e.g., radionuclides and their associated substances released from FNPP): (1) spatial relationship, (2) temporal relationship, (3) direct exposure, (4) phenotypic variability and spectrum, (5) experimental reproduction (external exposure), and (6) experimental reproduction (internal exposure) [10]. To our knowledge, the pale grass blue butterfly is the sole organism that has met the postulates in the case of the FNPP accident.

The second objective of our research is to understand the mechanisms of the biological effect caused by the FNPP accident. Mechanistic research has just begun in our laboratory, but this objective will be the focus of our laboratory for years to come.

The third objective of our research is to establish a system to monitor humanliving environments throughout Japan and around the world in the future using the pale grass blue butterfly and other similar butterfly species [11-14]. In Japan, following a future nuclear accident or large-scale chemical pollution event, the pale grass blue butterfly around an accident site may be collected, and their data may be readily compared with the reference data set already available [11, 12]. In Asia, Australia, and Africa, there are butterflies similar to the pale grass blue butterfly, and they can be used as indicator species $[13,14]$. 


\subsection{Field Surveys and Experiments in 2011}

In this section, we briefly review our first paper on the biological effect of the FNPP accident published in 2012 [8]. We first performed a field sampling attempt in early May 2011 to catch the first adult generation within the contaminated localities (where people continued to live) after the FNPP accident. We made another field sampling attempt in September 2011 for comparison.

We first measured the forewing size of collected butterflies. Size reduction was observed in the May male samples only. The male forewing size was inversely correlated with the ground radiation dose. Morphological abnormalities were checked for all samples; the abnormality rate was not high in May but increased in September. In September, the abnormality rate was highly correlated with the ground radiation dose.

We obtained the offspring $\left(\mathrm{F}_{1}\right)$ generation from morphologically normal female individuals caught at various localities. Their eclosion time (defined as the number of days from the first egg deposition to eclosion) and abnormality rate of appendages were both inversely correlated with the distance from FNPP. We further obtained the subsequent $\left(\mathrm{F}_{2}\right)$ generation. Abnormality rate of the $\mathrm{F}_{2}$ generation was relatively high, and similar types of abnormalities with the $\mathrm{F}_{1}$ generation were observed, suggesting the inheritance of abnormal traits through genetic damage.

We then used the pale grass blue butterfly from Okinawa (approximately $1,700 \mathrm{~km}$ southwest of FNPP and located at the southernmost tip of Japan), the least contaminated locality in Japan. An external exposure experiment using a cesium-137 $\left({ }^{137} \mathrm{Cs}\right)$ radiation source resulted in the smaller forewing size and dose-dependent survival curves. We also performed an internal exposure experiment, in which contaminated leaves from several localities were fed to larvae. Again, we observed the smaller forewing size and dose-dependent survival curves.

Together, the biological effect was demonstrated in the field samples, in the reared generations, and in the experimental reproductions. When this study was published, we received a large number of comments and criticism from around the world. Responses to these comments and criticism have been compiled, together with more detailed data analyses [15]. In that paper, we also newly presented the normal specimens of the pale grass blue butterfly caught before the FNPP accident. A thought-provoking discussion on this issue is also found in a commentary paper from other experts [16].

\subsection{Additional Exposure Experiments}

We quantitatively analyzed the amount of leaves and radioactive $\mathrm{Cs}$ that a larva ate in the internal exposure experiment [17]. Furthermore, we collected contaminated leaves with relatively low-level radioactive Cs from additional localities and per- 
formed additional internal exposure experiments [18]. As expected, we obtained dose-dependent mortality rates when larvae were fed the contaminated leaves. When the contaminated leaves were given to the next-generation larvae, the survival rate was low, but when noncontaminated leaves were given, the survival rate was high, despite the fact that their parents ate contaminated leaves. However, forewing size was inversely correlated with the cumulative amount of ingested radioactive Cs in two generations (parent and offspring).

The mortality and abnormality data that were obtained in these studies were compiled together and subjected to mathematical model fits [19]. The total abnormality data (including both mortality and abnormality) fit a Weibull function well; it is a sigmoidal curve used to describe a failure of mechanical or biological machinery. The data also fit a power function well.

Similarly, further external exposure experiments were performed. We collected contaminated soils, on which larvae from Okinawa were reared to simulate the external environment in contaminated localities. Again, we observed biological effects of external exposures (unpublished data).

\subsection{Field Surveys for 3 Years and the Japan-Wide Surveys}

The first study published in 2012 [8] contained the result of the field surveys that were performed in the spring and fall of 2011. We continued similar surveys twice a year until 2013 [20]. Generally, the total abnormality rate peaked in the fall of 2011 and the spring of 2012 and then decreased to normal levels by the fall of 2013. This decrease cannot solely be attributed to the decrease of contaminating radioactive Cs. The decrease probably indicates purifying selection against genetic mutants caused by the initial exposures from the FNPP accident. In addition, we revealed that the decrease in the total abnormality rate was, at least partially, a consequence of adaptive evolution of the pale grass blue butterfly to a contaminated environment [21]. The results indicate the importance of prompt surveys after nuclear (and probably other) accidents.

We also performed the Japan-wide surveys to understand the geographical distribution patterns of the forewing size with the help of Japanese amateur lepidopterists [22] because the forewing size decrease detected in the spring of 2011 should be evaluated in comparison with those from other parts of Japan. The northern populations at a high latitude had relatively small forewing sizes, but the border was located at the prefectural boundary between Fukushima and Miyagi Prefectures.

We further performed the Japan-wide surveys to understand the geographical distribution patterns of the abnormality rate and the wing color pattern modification rate $[11,12]$. There were no localities that had exceptionally high abnormality or modification rates except the northern populations. The northern populations had 
relatively high abnormality and modification rates, but the borders for these rates were located at the prefectural boundary between Miyagi and Iwate Prefectures.

In relation to the geographical borders of both the forewing size and the abnormality and modification rates, the Fukushima populations were categorized into the southern population. The fact that the smaller forewing size and the high abnormality rates were detected in the contaminated Fukushima populations after the FNPP accident cannot thus be attributed to natural phenotypic variation in the Fukushima populations.

\subsection{Internal Exposure Experiment Using the Cabbage White Butterfly}

The internal exposure experiments discussed above raised the question of whether this high sensitivity of the pale grass blue butterfly is simply an exceptional case. To answer this question, we used the cabbage white butterfly (Pieris rapae). Larvae of this butterfly eat cabbage leaves. We cultivated cabbage using contaminated soils collected from Fukushima to feed larvae from Okinawa. We observed morphological abnormalities in this butterfly [23]. Importantly, the percentage of granulocytes among hemocytes in this butterfly was inversely correlated with radioactive Cs concentration of cabbage leaves ingested but not with the potassium radioactivity concentration [23]. These results demonstrated that the high sensitivity of the pale grass blue butterfly to internal exposures was not exceptional. The results also indicated adverse cellular changes in hemocytes in response to ingested artificial radioactive Cs. The possibility of different biological consequences between radioactive potassium and radioactive $\mathrm{Cs}$ is also interesting.

\subsection{Toxicology of Cesium}

We evaluated the effect of nonradioactive Cs as a chemical substance by feeding larvae of the pale grass blue butterfly an artificial diet containing nonradioactive Cs chloride and demonstrated its low toxicity (unpublished data). Similarly, when radioactive $\mathrm{Cs}$ chloride $(\mathrm{CsCl})$ was fed via an artificial diet, its toxicity was also low in the pale grass blue butterfly (unpublished data), in comparison with the fieldcollected leaves discussed above. The difference may be called the field-laboratory paradox, but it can be explained by the contributions of the biologically mediated indirect field effect (see below). To the best of our knowledge, the pale grass blue butterfly is the sole organism that has both field-based and laboratory-based experimental results in addition to the field surveys and various types of examinations in the FNPP accident. 


\subsection{A 2016 Survey for Heavily Contaminated Localities}

In 2016, we performed a new field survey for heavily contaminated localities that were not included in our previous surveys. This survey was made possible because these localities have been made open to everybody since 2016 due to the completion of decontamination efforts; the Japanese government wants people to come back to the area. We found that the butterfly still showed the adverse effect of the accident in these localities in 2016 (unpublished data). Furthermore, the possible effect of radioactive iodine-131 $\left({ }^{131} \mathrm{I}\right)$ and ${ }^{137} \mathrm{Cs}$ for initial acute exposures was indicated statistically (unpublished data), suggesting that the major effect of initial radiation exposure was genetic damage.

\subsection{Detection of Genetic Damage}

Initial exposure by several radionuclides including ${ }^{131} \mathrm{I}$ and ${ }^{137} \mathrm{Cs}$ might have damaged DNA directly. As discussed above, genetic damage caused by the FNPP accident was first suggested by the rearing experiments for the $F_{1}$ and $F_{2}$ generations in 2011 [8]. Wing color pattern abnormalities in the field-caught and reared individuals who were phenotypically similar to those of genetic mutants also suggested genetic damage in the contaminated populations [6]. We also found that the adult abnormality rate was correlated well with the ground radiation dose using data points only from the contaminated localities surveyed in the fall of 2011 and the spring of 2012, suggesting that the abnormality peak at these time points are likely caused by the initial exposures that introduced genetic damage [24]. We have not published any molecular data to date, but we hope that we will be able to present them in the future.

One of the methods used to probe genome-wide DNA polymorphism is called amplified fragment-length polymorphism (AFLP) analysis. The genomic DNA was digested with restriction enzymes into numerous fragments, which were then amplified by polymerase chain reaction (PCR). Distribution patterns of the PCR fragment lengths were then analyzed by electrophoresis. We found that the abnormality rate was correlated with genetic diversity of the population and that genetic diversity was correlated with radiation dose (unpublished data). It appears that random mutations that were introduced by radiation exposure contributed to genetic diversity and then the abnormality rate.

Furthermore, the construction of the genome database for the pale grass blue butterfly, including the RNA-seq (whole transcriptome next generation sequencing) data for nontreated individuals, is under way. These data may be compared with the RNA-seq data from irradiated individuals to check for differences in gene expression patterns. 


\subsection{Overwintering Larvae and Dosimetric Analysis}

To evaluate the contribution of the initial exposures immediately after the FNPP accident to the biological impact quantitatively, it is important to calculate absorbed doses for the pale grass blue butterfly, considering both $\gamma$ - and $\beta$-rays [25]. Such a dosimetric analysis necessarily involves mathematical simulations that introduce many assumptions. To reduce the number of biological assumptions, it is important to understand the developmental stage of the pale grass blue butterfly and its surrounding environment at the time of the FNPP accident. We performed a field survey for overwintering larvae in March 2018, and we will continue this effort in 2019. Based on the larval size and environmental information, it will be possible to calculate the initial absorbed doses that larvae received, which may clarify the fieldlaboratory paradox to some extent.

\subsection{Field Effect via Biological Indirect Pathways}

The field-laboratory paradox may be solved by the field effect, which has been ignored by most radiation scientists. There may be several types of the field effect that are mediated by biological indirect pathways [24, 26-28]. For example, the host plant leaves may change their chemical components upon irradiation; toxic chemicals may be synthesized, or some important nutrients such as vitamins may not be synthesized. These chemical changes in host plants may cause death or abnormalities in larvae. Studies on the mechanisms of these pathways are now under way in the "model ecosystem" involving the pale grass blue butterfly and its host plant.

Acknowledgments The author is grateful to the editor, Prof. Fukumoto, for his invitation to this book project and for his comments on this article. The Fukushima Project at the BCPH Unit of Molecular Physiology was financially supported partially by the Incentive Project of the University of the Ryukyus, partially by the following private foundations, Takahashi Industrial and Economic Research Foundation, the Nohara Foundation, the Sumitomo Foundation, the Asahi Glass Foundation, and act beyond trust, and partially by public donors who kindly helped us to perform our study. The Fukushima Project was also supported by volunteer amateur lepidopterists and nonlepidopterists who provided invaluable samples (including butterflies and plants) and comments. The author also thanks the current and previous members of the BCPH Unit of Molecular Physiology for their contributions to the Fukushima Project.

\section{References}

1. Hiyama A, Iwata M, Otaki JM (2010) Rearing the pale grass blue Zizeeria maha (Lepidoptera, Lycaenidae): toward the establishment of a lycaenid model system for butterfly physiology and genetics. Entomol Sci 13:293-302

2. Otaki JM, Hiyama A, Iwata M et al (2010) Phenotypic plasticity in the range-margin population of the lycaenid butterfly Zizeeria maha. BMC Evol Biol 10:252 
3. Hiyama A, Taira W, Otaki JM (2012) Color-pattern evolution in response to environmental stress in butterflies. Front Genet 3:15

4. Buckley J, Bridle JR, Pomiankowski A (2010) Novel variation associated with species range expansion. BMC Evol Biol 10:382

5. Gilbert SF (2014) Developmental biology, 10th edn. Sinauer Associates, Sunderland

6. Iwata M, Hiyama A, Otaki JM (2013) System-dependent regulations of colour-pattern development: a mutagenesis study of the pale grass blue butterfly. Sci Rep 3:2379

7. Iwata M, Taira W, Hiyama A et al (2015) The lycaenid central symmetry system: color pattern analysis of the pale grass blue butterfly Zizeeria maha. Zool Sci 32:233-239

8. Hiyama A, Nohara C, Kinjo S et al (2012) The biological impacts of the Fukushima nuclear accident on the pale grass blue butterfly. Sci Rep 2:570

9. Hiyama A, Taira W, Sakauchi K et al (2018) Sampling efficiency of the pale grass blue butterfly Zizeeria maha (Lepidoptera: Lycaenidae): a versatile indicator species for environmental risk assessment. J Asia Pac Entomol 21:609-615

10. Taira W, Nohara C, Hiyama A et al (2014) Fukushima's biological impacts: the case of the pale grass blue butterfly. J Hered 105:710-722

11. Hiyama A, Taira W, Iwasaki M et al (2017) Geographical distribution of morphological abnormalities and wing color pattern modifications of the pale grass blue butterfly in northeastern Japan. Entomol Sci 20:100-110

12. Hiyama A, Taira W, Iwasaki M et al (2017) Morphological abnormality rate of the pale grass blue butterfly Zizeeria maha (Lepidoptera: Lycaenidae) in southwestern Japan: a reference data set for environmental monitoring. J Asia Pac Entomol 20:1333-1339

13. Gurung RD, Iwata M, Hiyama A et al (2016) Comparative morphological analysis of the immature stages of the grass blue butterflies Zizeeria and Zizina (Lepidoptera: Lycaenidae). Zool Sci 33:384-400

14. Iwata M, Matsumoto-Oda A, Otaki JM (2018) Rearing the African grass blue butterfly Zizeeria knysna: toward the establishment of a bioindicator in African countries. Afr Study Monogr 39:69-81

15. Hiyama A, Nohara C, Taira W et al (2013) The Fukushima nuclear accident and the pale grass blue butterfly: evaluating biological effects of long-term low-dose exposures. BMC Evol Biol 13:168

16. Møller AP, Mousseau TA (2013) Low-dose radiation, scientific scrutiny, and requirements for demonstrating effects. BMC Biol 11:92

17. Nohara C, Hiyama A, Taira W et al (2014) The biological impacts of ingested radioactive materials on the pale grass blue butterfly. Sci Rep 4:4946

18. Nohara C, Taira W, Hiyama A et al (2014) Ingestion of radioactively contaminated diets for two generations in the pale grass blue butterfly. BMC Evol Biol 14:193

19. Taira W, Hiyama A, Nohara $C$ et al (2015) Ingestional and transgenerational effects of the Fukushima nuclear accident on the pale grass blue butterfly. J Radiat Res 56:i2-i8

20. Hiyama A, Taira W, Nohara C et al (2015) Spatiotemporal abnormality dynamics of the pale grass blue butterfly: three years of monitoring (2011-2013) after the Fukushima nuclear accident. BMC Evol Biol 15:15

21. Nohara C, Hiyama A, Taira W et al (2018) Robustness and radiation resistance of the pale grass blue butterfly from radioactively contaminated areas: a possible case of adaptive evolution. J Hered 109:188-198

22. Taira W, Iwasaki M, Otaki JM (2015) Body size distributions of the pale grass blue butterfly in Japan: size rules and the status of the Fukushima population. Sci Rep 5:12351

23. Taira W, Toki M, Kakinohana K, Sakauchi K, Otaki JM (2019) Developmental and hemocytological effects of ingesting Fukushima's radiocesium on the cabbage white butterfly Pieris rapae. Sci Rep 9:2625

24. Otaki JM, Taira W (2018) Current status of the blue butterfly in Fukushima research. J Hered 109:178-187 
25. Endo S, Tanaka K, Kajimoto T et al (2014) Estimation of $\beta$-ray dose in air and soil from Fukushima Daiichi Power Plant accident. J Radiat Res 55:476-483

26. Garnier-Laplace J, Geras'kin S, Delta-Vedova C et al (2013) Are radiosensitivity data derived from natural field conditions consistent with data from controlled exposures? A case study of Chernobyl wildlife chronically exposed to low dose rates. J Environ Radioact 121:12-21

27. Otaki JM (2016) Fukushima's lessons from the blue butterfly: a risk assessment of the human living environment in the post-Fukushima era. Integr Environ Assess Manag 12:667-672

28. Bréchignac $\mathrm{F}$ (2016) The need to integrate laboratory- and ecosystem-level research for assessment of the ecological impact of radiation. Integr Environ Assess Manag 12:673-676

Open Access This chapter is licensed under the terms of the Creative Commons Attribution 4.0 International License (http://creativecommons.org/licenses/by/4.0/), which permits use, sharing, adaptation, distribution and reproduction in any medium or format, as long as you give appropriate credit to the original author(s) and the source, provide a link to the Creative Commons license and indicate if changes were made.

The images or other third party material in this chapter are included in the chapter's Creative Commons license, unless indicated otherwise in a credit line to the material. If material is not included in the chapter's Creative Commons license and your intended use is not permitted by statutory regulation or exceeds the permitted use, you will need to obtain permission directly from the copyright holder.

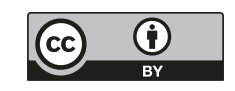

